\title{
Kripke semantics for modal bilattice logic
}

\author{
Achim Jung \\ School of Computer Science \\ University of Birmingham \\ Egbaston, Birmingham B15 2TT \\ United Kingdom \\ Email: a.jung@cs.bham.ac.uk
}

\author{
Umberto Rivieccio \\ School of Computer Science \\ University of Birmingham \\ Egbaston, Birmingham B15 2TT \\ United Kingdom \\ Email: u.rivieccio@cs.bham.ac.uk
}

\begin{abstract}
We employ the well-developed and powerful techniques of algebraic semantics and Priestley duality to set up a Kripke semantics for a modal expansion of Arieli and Avron's bilattice logic, itself based on Belnap's four-valued logic. We obtain soundness and completeness of a Hilbert-style derivation system for this logic with respect to four-valued Kripke frames, the standard notion of model in this setting. The proof is via intermediary relational structures which are analysed through a topological reading of one of the axioms of the logic. Both local and global consequence on the models are covered.
\end{abstract}

\section{INTRODUCTION}

Combining multi-valued and modal logics into a single system is a long-standing concern in mathematical logic and computer science, see for example [12], [13] and the literature cited there. Given the necessity for multiple truth values in many areas of computer science and AI, on the one hand, and the spectacular success of applied modal calculi in verification, on the other, the justification for studying combined calculi is as strong as ever.

Similarly, it is widely accepted that Kripke frames provide a semantics for modal logics that is both flexible with regards to intended applications and interpretations, and highly intuitive. When the non-modal part is multi-valued, though, one may wonder whether the accessibility relation between worlds should remain two-valued or itself be allowed to assume the same range of truth values as the logic itself. Starting from the point of view of AI applications, Fitting [12], [13] argues that multiple values are an appropriate and useful modelling device, and this is the approach taken here, too. Also, restricting to Kripke structures with two-valued accessibility relations corresponds to a strengthening of the logic, contrary to our interest in identifying the minimal modal logic.

Our logic is four-valued and thus in the tradition of bilattice logic begun by Belnap [2], [3] and subsequently greatly extended by Arieli and Avron [1] and others. The additional values besides the classical t ("true") and f ("false") are $\perp$ (for "unknown") and $T$ (for "contradiction") with obvious interpretations and applications in a computer science setting. For the present purposes it is noteworthy that bilattice logic makes use of two designated truth values, $\mathrm{t}$ and $T$ and that furthermore $\neg \top=\top$, that is, negation does not convert all designated values to non-designated ones. Much of the existing literature on multi-valued logics (modal or otherwise) is therefore not immediately applicable. Furthermore, the Belnap lattice, when seen as a residuated lattice, is not integral (in a sense explained below) which sets it apart from the tradition in multi-valued logic. Finally, modal expansions of bilattice logic are not normal in the sense that the axiom $\square(\varphi \rightarrow \psi) \rightarrow(\square \varphi \rightarrow \square \psi)$ is not valid. This implies that the standard techniques of modal logic are not applicable without close scrutiny and adaptation. To summarise, modal bilattice logic is natural and desirable yet much of the existing literature does not apply to it. It is the purpose of the present paper to show that nevertheless a very attractive and satisfactory Kripke semantics can be established.

Our overall strategy can be summarised by the following diagram:

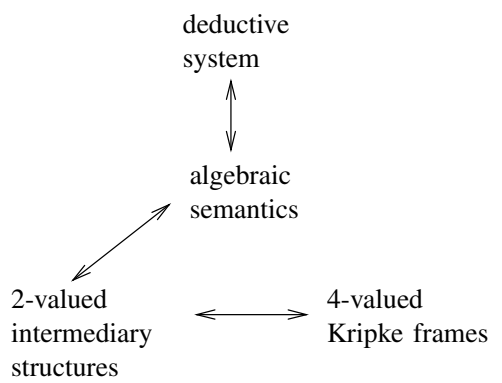

The link at the top will allow us to exploit the rich body of knowledge concerning bilattices, and we will make use of [25], [20] and [21]. Since the "local" version of the consequence relation we study is not algebraizable (in the sense of [5]), establishing this link is one of the results of this paper. The link on the left combines Jónsson-Tarski duality for modal algebras with the general duality for bilattices established in [18] (drawing on results in [22]). The link at the bottom, finally, is established by a careful analysis of the 2-valued intermediary structures that arise from duality, interpreting and exploiting the axiom $\square(\perp \rightarrow \varphi) \leftrightarrow(\perp \rightarrow \square \varphi)$.

Outline. As we have explained, much of the existing literature on modal multi-valued logics does not apply to modal bilattice logic, and so in the present paper we take care to explain the setting at every stage, pointing out similarities, differences, and alternative approaches. In Section II we introduce the language of bilattice logic and explain the choices of basic connectives available in it. This provides the background for our discussion of four-valued Kripke structures in the following section and the evaluation of the 
$\square$-operator in particular. In the second part of Section III we present a Hilbert-style axiomatisation of modal bilattice logic, extending the one given by Arieli and Avron in [1] for the non-modal part. Both local and global consequence are covered. In Section IV we start on our completeness proof by providing an algebraic semantics in modal bilattices. These structures are further analysed in the following section, where we show that they can be seen as twist-structures constructed from bimodal Boolean algebras. This is important for the the rest of the paper but it also allows us to conclude that our logic properly subsumes that of [24]. In Section VI we show how Priestley duality for bilattices and Jónsson-Tarski duality for modal algebras can be combined to obtain a duality between modal bilattices and modal bispaces. We have then all the tools ready to prove completeness in Section VII. A key ingredient of the completeness proof is the fact that modal bispaces give rise to four-valued Kripke structures. We discuss our results in Section VIII and indicate lines for further research.

\section{Algebraic PREliminaries}

The non-modal fragment of the logics we are going to study is the multi-valued logic of Arieli and Avron [1] (henceforth referred to as bilattice logic or Arieli-Avron logic), which arose from Belnap's famous proposal for a "useful four-valued logic" [3]. Belnap saw that his four truth values can be arranged as lattices in two distinct but equally meaningful ways, ordering them either by information content (the knowledge order $\leq_{k}$ ) or by logical strength (the truth order $\leq_{t}$ ), see Figure 1.
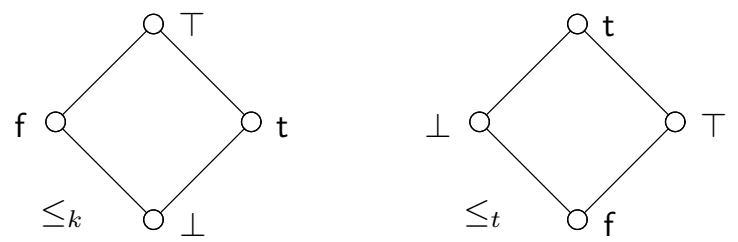

Fig. 1. The four-element Belnap bilattice $\mathcal{F O U} \mathcal{R}$ in its two orders

Generally, a (bounded) bilattice is an algebra $\langle B, \wedge, \vee, \otimes, \oplus, \neg, \mathrm{f}, \mathrm{t}, \perp, \top\rangle$ such that $\langle B, \wedge, \vee, \mathrm{f}, \mathrm{t}\rangle$ and $\langle B, \otimes, \oplus, \perp, \top\rangle$ are both bounded lattices. The order $\leq_{t}$ arising from $\wedge$ or $\vee$ is called the truth order, that arising from $\otimes$ or $\oplus$ the knowledge order $\leq_{k}$. The negation operation $\neg$ is required to satisfy the properties

(i) $x \leq_{t} y$ iff $\neg y \leq_{t} \neg x$;

(ii) $x \leq_{k} y$ iff $\neg x \leq_{k} \neg y$;

(iii) $\neg \neg x=x$,

which on $\mathcal{F O U R}$ determine negation uniquely: $\neg \mathrm{t}=\mathrm{f}, \neg \mathrm{f}=$ $\mathrm{t}, \neg \top=\top$, and $\neg \perp=\perp$. From a logical point of view, $\wedge$ and $\otimes$ are interpreted as conjunctions, and $\vee$ and $\oplus$ as disjunctions.

Just as the notion of (ultra)filter is fundamental in the algebraic study of classical logic, a key notion for the logical study of bilattices is that of bifilter [1, Definition 2.13]. A bifilter of a bilattice $\mathbf{B}$ is a non-empty set $F \subseteq B$ that is a lattice filter with respect to both lattice orders. A bifilter is prime if it is a prime filter with respect to both orders. Every bilattice has a least bifilter $F_{0}$, which is generated by the set $\{T, \mathrm{t}\}$.

The four-element algebra $\mathcal{F} \mathcal{O U} \mathcal{R}$, having $\{\mathrm{f}, \mathrm{t}, \perp, \top\}$ as its carrier set, is the smallest non-trivial bilattice. Here, the least bifilter $F_{0}=\{\top, \mathrm{t}\}$ is the only proper one; it is also prime.

In addition to the algebraic operations of bilattices, two implication operations are naturally available on $\mathcal{F} \mathcal{O U} \mathcal{R}$. Weak implication $\supset[1$, Definition 3.15] is defined by

$$
x \supset y:= \begin{cases}\mathrm{t} & \text { if } x \notin F_{0} \\ y & \text { if } x \in F_{0}\end{cases}
$$

Strong implication $\rightarrow[1$, Definition 3.25] is defined by the term $x \rightarrow y:=(x \supset y) \wedge(\neg y \supset \neg x)$. The choice of this terminology is justified by the fact that each of these operations shares some of the logical properties of classical (i.e., Boolean) implication. For instance, Arieli-Avron logic enjoys the classical deduction theorem with respect to weak implication [1, Proposition 3.16]. On the other hand, strong implication has a better interaction with negation. For example, it satisfies the contraposition law $(\varphi \rightarrow \psi$ is inter-derivable with $\neg \psi \rightarrow \neg \varphi$ ) and determines the truth lattice order of $\mathcal{F O U R :}$

$$
\begin{array}{lll}
x \leq_{t} y & \text { iff } & x \rightarrow y \in F_{0} \\
& \text { iff } \quad x \rightarrow y=(x \rightarrow y) \rightarrow(x \rightarrow y) .
\end{array}
$$

Another observation which will play a key role in our study of modal bilattice logics is that $\mathcal{F O U R}$ can be viewed as a residuated lattice. This means that by setting $x * y:=\neg(y \rightarrow \neg x)$ we obtain a commutative monoid $\langle\mathcal{F} \mathcal{O} \mathcal{R}, *, \top\rangle$ and the following property (residuation) is satisfied [25, Proposition 5.4.1]:

$$
x * y \leq_{t} z \quad \text { iff } \quad y \leq_{t} x \rightarrow z .
$$

Residuated lattices are well-known in algebraic logic as they provide algebraic semantics for a wide class of multi-valued logics, including the so-called fuzzy logics [16].

The truth tables of the adjoint pair $\langle *, \rightarrow\rangle$ in $\mathcal{F O U} \mathcal{R}$ are displayed below:

\begin{tabular}{c|c|c|c|c}
$*$ & $\mathrm{f}$ & $\perp$ & $\mathrm{t}$ & $\mathrm{t}$ \\
\hline $\mathrm{f}$ & $\mathrm{f}$ & $\mathrm{f}$ & $\mathrm{f}$ & $\mathrm{f}$ \\
\hline$\perp$ & $\mathrm{f}$ & $\mathrm{f}$ & $\perp$ & $\perp$ \\
\hline$T$ & $\mathrm{f}$ & $\perp$ & $\top$ & $\mathrm{t}$ \\
\hline $\mathrm{t}$ & $\mathrm{f}$ & $\perp$ & $\mathrm{t}$ & $\mathrm{t}$
\end{tabular}

\begin{tabular}{c|c|c|c|c}
$\rightarrow$ & $\mathrm{f}$ & $\perp$ & $\mathrm{T}$ & $\mathrm{t}$ \\
\hline $\mathrm{f}$ & $\mathrm{t}$ & $\mathrm{t}$ & $\mathrm{t}$ & $\mathrm{t}$ \\
\hline$\perp$ & $\perp$ & $\mathrm{t}$ & $\perp$ & $\mathrm{t}$ \\
\hline $\mathrm{T}$ & $\mathrm{f}$ & $\perp$ & $\mathrm{T}$ & $\mathrm{t}$ \\
\hline $\mathrm{t}$ & $\mathrm{f}$ & $\perp$ & $\mathrm{f}$ & $\mathrm{t}$
\end{tabular}

In the same way as we think of $\supset$ and $\rightarrow$ as implications, one can view $*$ as yet another conjunction, called multiplicative or strong conjunction in the literature on multi-valued logics. Note, though, that $*$ is not idempotent, so it does not define a third ordering on $\mathcal{F O U} \mathcal{R}$.

Altogether, then, we view $\mathcal{F} \mathcal{O U} \mathcal{R}$ not just as a bilattice, but as an algebra in the enriched language that includes weak implication (and therefore also the term-definable $\rightarrow$ and $*$ ):

Definition 1. The class of (bounded) classical implicative bilattices is the variety generated by $\mathcal{F O U R}$ in the algebraic language $\langle\wedge, \vee, \otimes, \oplus, \supset, \neg, \mathrm{f}, \mathrm{t}, \perp, \top\rangle$. 
This variety, axiomatized in [25, Definition 4.3.1], provides an algebraic semantics (in fact, the equivalent algebraic semantics in the sense of [5]) of the Arieli-Avron calculus [1, Definition 3.15]; this fact is proved in [25, Theorem 4.3.10]. We also refer to [25, Ch. 4 and 5] for further details and proofs concerning the results on Arieli-Avron logic that we will use in the next sections.

\section{MOdAL EXPANSIONS OF BILATTICE LOGIC}

\section{A. Semantics}

Algebraically, Arieli-Avron logic is defined as the logic of the variety of classical implicative bilattices with bifilters as their set of designated truth values. The same logic arises when one restricts the bifilters to prime ones [25] or even when one just considers $\mathcal{F} \mathcal{O U} \mathcal{R}$ together with $F_{0}=\{\mathrm{t}, \top\}$ [1]. For our purposes it is most convenient to adopt the last alternative.

Let $\mathbf{F m}$ be the formula algebra over the language $\langle\wedge, \vee, \otimes, \oplus, \supset, \neg, \mathrm{t}, \mathrm{f}, \top, \perp\rangle$, freely generated by a countable set of variables Var. For $\Gamma \cup\{\varphi\} \subseteq F m$ (the carrier set of $\mathbf{F m}$ ), we define $\Gamma \vDash \varphi$ if and only if, for all homomorphisms $h: \mathbf{F m} \rightarrow \mathcal{F O U R}$, if $h(\gamma) \in F_{0}=\{\mathrm{t}, \top\}$ for all $\gamma \in \Gamma$, then $h(\varphi) \in F_{0}$. This is the non-modal core of our logic. ${ }^{1}$

For a modal expansion of this logic we initially focus on the necessity operator $\square$. Semantically, we seek to interpret it in suitable Kripke structures. For motivation, let us consider first a classical Kripke model $\langle W, R, v\rangle$, where $W$ is a set of "worlds", $R$ an accessibility relation among them and $v$ a valuation. Now view $R$ as the characteristic function associated with the accessibility relation, i.e., as a map $R: W \times W \rightarrow\{\mathrm{t}, \mathrm{f}\}$. Similarly, view $v: F m \times W \rightarrow\{\mathrm{t}, \mathrm{f}\}$ as a map assigning to each formula $\varphi \in F m$ at each point $w \in W$ a truth value in $\{t, f\}$. By the so-called standard translation of modal logic into first-order logic, we obtain the following definition for the semantics of the necessity operator

$$
v(\square \varphi, w):=\bigwedge\left\{R\left(w, w^{\prime}\right) \rightarrow v\left(\varphi, w^{\prime}\right): w^{\prime} \in W\right\}
$$

where $\Lambda$ denotes the infinitary meet corresponding to the universal quantifier and $\rightarrow$ is Boolean implication. ${ }^{2}$

This definition can now easily be adapted to our four-valued setting. We consider Kripke models $\langle W, R, v\rangle$ where both $R$ and $v$ are four-valued, that is, we define $R: W \times W \rightarrow \mathcal{F O U R}$ and $v: F m \times W \rightarrow \mathcal{F O U R}$. As before, valuations are required to be homomorphisms in their first argument. We stress, as this will be important for our axiomatization, that we have included the constants $t, f, \top, \perp$ in the propositional language, so valuations must interpret each of them as the corresponding element of $\mathcal{F O U R}$.

Since $\mathcal{F O U R}$ carries three distinct conjunctions and two implications there are six candidates for the translations of (3) to the four-valued setting. We reject the monoid operation $*$

\footnotetext{
${ }^{1}$ Note that, by its very definition, this logic is a conservative expansion of Belnap-Dunn logic [2], [14] ( $\supset$ being the additional connective).

${ }^{2}$ Note that conjunction is taken in the complete lattice of truth values, so there is no problem with using the infinitary operation $\Lambda$.
}

because it is not idempotent and hence would require us to replace the set $\left\{R\left(w, w^{\prime}\right) \rightarrow v\left(\varphi, w^{\prime}\right): w^{\prime} \in W\right\}$ by a multi-set. The choice between $\wedge$ and $\otimes$ is more subtle as relates to the intended interpretation of the necessity operator. Our choice is for the "logical" connective rather than the knowledge order one as it is here that there are useful interactions with the two implications. This leaves the pairs $\langle\wedge, \rightarrow\rangle$ and $\langle\wedge, \supset\rangle$.

The latter choice has the disadvantage (in our opinion) that the accessibility relation $R$, although formally introduced as four-valued, turns out to have a two-valued behaviour when interacting with weak implication. This is so because in $\mathcal{F O U R}$ the value of (3) (with $\rightarrow$ replaced by $\supset$ ) is the same as the following one:

$$
\bigwedge\left\{v\left(\varphi, w^{\prime}\right): R\left(w, w^{\prime}\right) \in F_{0}\right\} .
$$

In fact, the choice $\langle\wedge, \supset\rangle$ has already been considered in [24] to introduce a modal expansion of Belnap-Dunn logic (that is, the $\{\wedge, \vee, \neg, f, t\}$-fragment of Arieli-Avron bilattice logic). It turns out, however, that the resulting operator is strictly less expressive than the one defined by the pair $\langle\wedge, \rightarrow\rangle$. Let us denote the two choices by $\square_{\supset}$ and $\square_{\rightarrow}$. We get:

Proposition 2. For all formulas $\varphi \in F m$, all four-valued Kripke models $\langle W, R, v\rangle$, and all $w \in W$ :

$$
v\left(\square_{\supset} \varphi, w\right)=v\left(\square_{\rightarrow}(\varphi \vee \perp) \oplus\left(\square_{\rightarrow} \varphi \wedge \perp\right), w\right) .
$$

One may wonder whether, conversely, it is possible to define $\square_{\rightarrow}$ from $\square_{\supset}$. This is already unlikely given the twovalued nature of the latter, and our algebraic analysis below (Section V) will indeed confirm this intuition.

To summarise, our choice for the semantics of the necessity operator is based on the pair $\langle\wedge, \rightarrow\rangle$, that is, in the four-valued context we replace classical conjunction with the truth lattice meet and classical implication with the strong implication of Arieli-Avron logic. From now on we will write simply $\square$ in place of $\square_{\rightarrow}$.

Let us point out a further pleasing feature of $\square$. Given that $\mathcal{F O U R}$ can be seen as a residuated lattice, and since strong implication interacts well with negation, we can define a possibility operator $\diamond$ which will turn out in the logic to be dual to $\square$. Semantically, it is given by [6, p.746]:

$$
v(\diamond \varphi, w):=\bigvee\left\{R\left(w, w^{\prime}\right) * v\left(\varphi, w^{\prime}\right): w^{\prime} \in W\right\} .
$$

This is obviously a generalization of the classical definition with the monoid operation replacing classical conjunction. ${ }^{3}$

We are now ready to extend the semantic consequence relation of Arieli-Avron logic to our modal expansion. We say that a point $w \in W$ of a four-valued model $M=\langle W, R, v\rangle$ satisfies a formula $\varphi \in F m$ if $v(\varphi, w) \in\{\mathrm{t}, \top\}$. In such a case we write $M, w \vDash \varphi$. For a set of formulas $\Gamma \subseteq F m$, we write $M, w \vDash \Gamma$ to mean that $M, w \vDash \gamma$ for each $\gamma \in \Gamma$. As is usual in modal logic, we consider two consequence relations. The local consequence $\Gamma \vDash_{l} \varphi$ holds if for every

\footnotetext{
${ }^{3}$ As we noted before, $*$ is not idempotent but this is not a problem here as it is applied to two terms, not to a set.
} 
model $M=\langle W, R, v\rangle$ and every $w \in W$, it is the case that $M, w \vDash \Gamma$ implies $M, w \vDash \varphi$. The global consequence relation $\Gamma \vDash_{g} \varphi$ holds if, for every model $M$, if $M, w \vDash \Gamma$ for all $w \in W$, then $M, w \vDash \varphi$ for all $w \in W$.

We remind the reader that the above definitions imply that:

- if $\Gamma \vDash_{l} \varphi$, then $\Gamma \vDash_{g} \varphi$ (global consequence is a strengthening of the local one);

- $\emptyset \vDash_{l} \varphi$ if and only if $\emptyset \vDash_{g} \varphi$ (the two consequences have the same valid formulas).

Let us now explore the axioms and rules that are valid semantically.

Proposition 3. The following formulas are valid in all models: ${ }^{4}$

(i) $\square \mathrm{t} \leftrightarrow \mathrm{t}$

(ii) $\square(\varphi \wedge \psi) \leftrightarrow(\square \varphi \wedge \square \psi)$,

(iii) $\square(\mathrm{c} \rightarrow \varphi) \leftrightarrow(\mathrm{c} \rightarrow \square \varphi)$ for all $\mathrm{c} \in\{\mathrm{t}, \mathrm{f}, \top, \perp\}$

As in [6], the last of these schemata will play a prominent role in the axiomatization of our logic, as will the following rule:

Proposition 4 (Monotonicity). The rule $\varphi \rightarrow \psi \vdash \square \varphi \rightarrow \square \psi$ is sound with respect to global consequence. In other words, $\varphi \rightarrow \psi \vDash_{g} \square \varphi \rightarrow \square \psi$ holds.

The following is an immediate consequence of monotonicity:

Corollary 5. If $\varphi \rightarrow \psi$ is a formula valid in all models then so is $\square \varphi \rightarrow \square \psi$.

However, necessitation $\varphi \vDash_{g} \square \varphi$, which classically is equivalent to monotonicity, is not sound. (This is a consequence of the fact that $F_{0}$ is not an exponential ideal of $\mathcal{F O U R}$, i.e., $x \in F_{0}$ does not imply $a \rightarrow x \in F_{0}$.) The normality axiom, $\square(\varphi \rightarrow \psi) \rightarrow(\square \varphi \rightarrow \square \psi)$, also fails and it is easy to find small counter-models to it (or see [6]). Thus, the modal logic we are studying is non-normal. This constitutes one of the main difficulties in axiomatizing it, as the standard canonical model construction cannot be applied to prove completeness.

In light of these negative results is rather fortuitous that the monotonicity rule holds and this may give further credence to our choice of the semantics of $\square$.

\section{B. Axiomatizations}

In this section we introduce Hilbert-style calculi which we will prove to be complete with respect to the global and the local consequence relations, respectively. Our starting point is the axiomatization of the non-modal fragment of our logic provided by Arieli and Avron [1, p. 47]. We present the axiom schemata in stages:

$$
\begin{aligned}
& \text { (つ 1) } \quad p \supset(q \supset p) \\
& (\supset \text { 2) } \quad(p \supset(q \supset r)) \supset((p \supset q) \supset(p \supset r)) \\
& (\supset 3) \quad((p \supset q) \supset p) \supset p \\
& (\neg \neg) \quad p \supset \neg \neg p \quad \neg \neg p \supset p
\end{aligned}
$$

\footnotetext{
${ }^{4}$ We use $\varphi \leftrightarrow \psi$ as a shorthand for $(\varphi \rightarrow \psi) \wedge(\psi \rightarrow \varphi)$.
}

Note that the usual schema $(\neg p \supset \neg q) \supset(q \supset p)$ (contraposition) is absent but the classical nature of the calculus has been preserved by the inclusion of Peirce's Law ( $\supset$ 3) and double negation. ${ }^{5}$ The next set of schemata establishes the link with the truth lattice operations and is entirely standard:

$$
\begin{array}{lll}
(\wedge \supset) & (p \wedge q) \supset p & (p \wedge q) \supset q \\
(\supset \wedge) & p \supset(q \supset(p \wedge q)) & \\
(\supset \mathrm{t}) & & p \supset \mathrm{t} \\
(\supset \vee) & p \supset(p \vee q) & q \supset(p \vee q) \\
(\vee \supset) & (p \supset r) \supset((q \supset r) \supset((p \vee q) \supset r)) \\
(\supset \mathrm{f}) & \mathrm{f} \supset p &
\end{array}
$$

The analogous schemata for the information lattice operations:

$$
\begin{array}{lll}
(\otimes \supset) & (p \otimes q) \supset p & (p \otimes q) \supset q \\
(\supset \otimes) & p \supset(q \supset(p \otimes q)) & \\
(\supset \top) & p \supset \top & \\
(\supset \oplus) & p \supset(p \oplus q) & q \supset(p \oplus q) \\
(\oplus \supset) & (p \supset r) \supset((q \supset r) \supset((p \oplus q) \supset r)) \\
(\supset \perp) & \perp \supset p &
\end{array}
$$

In the absence of contraposition one also has to stipulate how negation interacts with the other operations:

$$
\begin{array}{ll}
(\neg \wedge) & \neg(p \wedge q) \equiv(\neg p \vee \neg q) \\
(\neg \vee) & \neg(p \vee q) \equiv(\neg p \wedge \neg q) \\
(\neg \otimes) & \neg(p \otimes q) \equiv(\neg p \otimes \neg q) \\
(\neg \oplus) & \neg(p \oplus q) \equiv(\neg p \oplus \neg q) \\
(\neg \supset) & \neg(p \supset q) \equiv(p \wedge \neg q) \\
(\neg \mathrm{t}) & \neg \mathrm{t} \supset p \\
(\neg \mathrm{f}) & p \supset \neg \mathrm{f} \\
(\neg \top) & p \supset \neg \top \\
(\neg \perp) & \neg \perp \supset p
\end{array}
$$

where $\varphi \equiv \psi$ abbreviates the two schemata $\varphi \supset \psi$ and $\psi \supset \varphi$. The only rule of the Arieli-Avron calculus is modus ponens:

$$
\text { (mp) } \quad p, p \supset q \vdash q
$$

As is shown in [1], this calculus is complete with respect to the semantics based on $\mathcal{F O U R}$ presented at the beginning of Section III-A.

We now proceed to expand the Arieli-Avron calculus to accommodate the modal necessity operator, taking our cue from the semantic considerations in in the previous subsection. We begin by adding the axiom schemata

$$
\begin{array}{ll}
(\square \mathrm{t}) & \square \mathrm{t} \leftrightarrow \mathrm{t} \\
(\square \wedge) & \square(p \wedge q) \leftrightarrow(\square p \wedge \square q) \\
(\square \perp) & \square(\perp \rightarrow p) \leftrightarrow(\perp \rightarrow \square p)
\end{array}
$$

\footnotetext{
${ }^{5}$ In fact the $\{\wedge, \vee, \supset\}$ fragment of Arieli-Avron logic coincides with the negation-free fragment of classical logic.
} 
Interestingly, the last of these covers only one of the four cases that make up Proposition 3-(iii), and indeed, one of the consequences of our completeness result is that the other three are not needed. In order to capture the closure property expressed in Corollary 5 we need to make sure that we first generate all valid instances of the shape $\varphi \rightarrow \psi$. The official definition of our logic is therefore slightly more involved than usual:

Definition 6. Let Fm be the set of formulas generated by a countable set of variables $V a r$ in the modal language $\langle\wedge, \vee, \otimes, \oplus, \supset, \neg, \mathrm{f}, \mathrm{t}, \perp, \top, \square\rangle$. The set $\Sigma$ of axioms of modal bilattice logic is the least subset of Fm containing all substitution instances of the schemata exhibited above, and closed under

(val-mp) if $\varphi$ and $\varphi \supset \psi$ are in $\Sigma$, then so is $\psi$; (val-mono) if $\varphi \rightarrow \psi$ is in $\Sigma$, then so is $\square \varphi \rightarrow \square \psi$.

The rules of modal bilattice logic are

$$
\frac{\varphi, \varphi \supset \psi}{\psi} \text { (mp) } \frac{\varphi \rightarrow \psi}{\square \varphi \rightarrow \square \psi} \text { (mono) }
$$

Local inference $\vdash_{l}$ employs only (mp), while global inference $\vdash_{g}$ is generated by (mp) and (mono). ${ }^{6}$

Having defined calculi for both local and global consequence on four-valued Kripke-structures, we will now embark on the completeness proof. Our strategy for doing so is fairly different from that of [6]. The latter is in a certain sense more general than ours, as it applies to any finite integral residuated lattice. Ours, however, is not a special case of [6] because their proof only applies to residuated lattices which are integral, that is, ones where the identity of the monoid operation $*$ coincides with the maximum element of the associated lattice order; this does not hold in $\mathcal{F O U R}$ because $T$ is the monoid identity but $t$ is the maximum element of the truth order. Moreover, the proof of [6] is rather syntactical (see, e.g., [6, Claims 4.9 and 4.10]) and because of this, in our opinion, less transparent than the ones we will present. We use instead algebra and topology, so that our strategy is more indirect but also more structural. As it turns out, it is also more powerful in the sense that it allows us to prove completeness also for $\vdash_{g}$, a problem which is left open in [6].

Our strategy is as follows. We first prove that both our modal consequence relations enjoy algebraic completeness with respect to a variety of bilattices expanded with a modal operator, the difference being in the sets of designated elements that are allowed. As happens with classical modal logic, the global consequence relation is in fact algebraizable [5], whereas the local one is only complete with respect to this algebra-based semantics.

\footnotetext{
${ }^{6}$ Note that, although structurally similar, the rules (val-mp) and (valmono) are only ever applied to valid formulas, while (modus ponens) and (monotonicity) can be applied to arbitrary assumptions.
}

We then develop a topological duality theory for modal bilattices which will allow us to construct a generalized (two-valued) relational semantics for our logics; these are the intermediary structures mentioned in the Introduction. Finally, we show that intermediary structures can be used to build four-valued Kripke (counter)-models and thus to prove completeness of our calculi with respect to the semantics introduced in III-A.

\section{Algebraic completeness}

Algebraic logic, begun by Tarski in 1935 [27], is by now a well-developed methodology for the study of derivation systems, and particularly well-suited for propositional and modal calculi. A convenient reference for our purposes is [5]; see also [10], [15]. In a nutshell, the idea is to translate the axioms and rules of a deductive system into the equational logic of a suitable class of algebras. The strongest correspondence of algebraic logic is that of algebraic equivalence and it holds when the deductive system and the equational logic can be mutually translated into each other, see [5, Definition 2.8]. The logic in this case is called algebraizable. One of the consequences of algebraizability is that a complete algebraic semantics (with matrices, that is, algebras and sets of designated elements) can be constructed mechanically from the deductive system via the so-called defining equations and equivalence formulas which underlie the two translations, see [5, Theorem 2.17].

Arieli's and Avron's bilattice logic was shown to be algebraizable by the second author in [25]. The corresponding algebraic semantics is the variety of classical implicative bilattices (Definition 1) with defining equation $\varphi=\varphi \supset \varphi$ and equivalence formula $\varphi \leftrightarrow \psi$. The set of designated elements for a classical implicative bilattice is exactly the smallest bifilter (which is the one generated by $\{\mathrm{t}, \top\}$ ).

In more detail, let $\vdash$ denote the derivability relation of Arieli's and Avron's calculus, and let $\Gamma \cup\{\varphi\} \subseteq F m$. We have that $\Gamma \vdash \varphi$ iff, for every pair $\left\langle\mathbf{B}, F_{0}\right\rangle$, where $\mathbf{B}$ is a classical implicative bilattice $\mathbf{B}$ and $F_{0}$ is the least bifilter of $\mathbf{B}$, and for all homomorphisms $h: \mathbf{F m} \rightarrow \mathbf{B}$, it holds that, if $h(\gamma) \in F_{0}$ for all $\gamma \in \Gamma$, then $h(\varphi) \in F_{0}$.

One of the striking features of algebraizability is that it is an intrinsic feature of a derivation system, [5, Theorem 4.7]. As can be seen from the conditions listed there, adding new connectives to a logic will often preserve algebraizability. This is the case here with the global inference system $\vdash_{g}$ for modal bilattice logic. The condition needed is exactly the monotonicity rule (mono). By the same token, local inference is not algebraizable.

Defining equation and equivalence formula remain the same in the modal expansion and using them to translate the additional axioms we obtain the axiomatization of the variety that is the basis for the complete algebraic semantics:

Definition 7. A modal bilattice is an algebra $\langle B, \wedge, \vee, \otimes, \oplus, \supset, \neg, \mathrm{f}, \mathrm{t}, \perp, \top, \square\rangle$ such that the reduct 
obtained by dropping $\square$ is a classical implicative bilattice and the following equations are satisfied: ${ }^{7}$

$$
\begin{aligned}
& \square \mathrm{t}=\mathrm{t} \\
& \square(x \wedge y)=\square x \wedge \square y
\end{aligned}
$$$$
\text { (iii) } \quad \square(\perp \rightarrow x)=\perp \rightarrow \square x \text {. }
$$

Returning to a comment we made above, we note that every modal bilattice satisfies the equation $\square(\mathrm{c} \rightarrow \varphi)=\mathrm{c} \rightarrow \square \varphi$ for each $c \in\{f, t, \perp, \top\}$. This can be shown purely algebraically and then translated back into the logic as $\square(\mathrm{c} \rightarrow \varphi) \leftrightarrow$ $(c \rightarrow \square \varphi)$.

To summarize our discussion:

Theorem 8. The global consequence relation $\vdash_{g}$ of modal bilattice logic is algebraizable with respect to the variety of modal bilattices, with defining equation $\varphi=\varphi \supset \varphi$ and equivalence formula $\varphi \leftrightarrow \psi$.

Theorem 9. The global consequence relation $\vdash_{g}$ is complete with respect to the class of all matrices $\left\langle\mathbf{B}, F_{0}\right\rangle$ such that $\mathbf{B}$ is a modal bilattice $\mathbf{B}$ and $F_{0}$ is the least bifilter of $\mathbf{B}$.

As happens with classical modal logic, the global and the local consequence relations share the same algebraic counterpart. However, $\vdash_{l}$ has looser relation with the algebraic semantics (in particular, it is not algebraizable). Nevertheless we are able to prove the following:

Theorem 10. The local consequence relation $\vdash_{l}$ is complete with respect to the class of all matrices $\langle\mathbf{B}, F\rangle$ such that $\mathbf{B}$ is a modal bilattice $\mathbf{B}$ and $F$ is a bifilter of $\mathbf{B}$.

Let us stress the difference with Theorem 9: now we need to consider arbitrary bifilters, not just the least one.

\section{Modal Bilattices as twist-Structures}

Several classes of bilattices can be conveniently represented through a construction called twist-structure [21], [7]. In this section we extend it to obtain a representation for modal bilattices, which will greatly enhance our understanding of the necessity operator $\square$ and also clarify the connection between our logic and that of [24].

Definition 11. A bimodal Boolean algebra is a structure $\mathbf{A}=\left\langle A, \sqcap, \sqcup, \sim, 0,1, \square_{+}, \square_{-}\right\rangle$such that $\langle A, \sqcap, \sqcup, \sim, 0,1\rangle$ is a Boolean algebra and both $\square_{+}$and $\square_{-}$are unary operators that preserve finite meets.

The above definition implies that both $\left\langle A, \sqcap, \sqcup, \sim, 0,1, \square_{+}\right\rangle$ and $\left\langle A, \sqcap, \sqcup, \sim, 0,1, \square_{-}\right\rangle$are modal Boolean algebras in the usual sense [9].

Given a bimodal Boolean algebra $\mathbf{A}$, we consider the twiststructure

$$
\mathbf{A}^{\bowtie}=\langle A \times A, \wedge, \vee, \otimes, \oplus, \supset, \neg, \mathrm{f}, \mathrm{t}, \perp, \top, \square\rangle
$$

\footnotetext{
${ }^{7}$ For those who are familiar with algebraic logic: The quasi-equation corresponding to the monotonicity rule holds as a consequence of $\square(x \wedge y)=$ $\square x \wedge \square y$ (monotonicity of $\square$ with respect to the truth lattice order).
}

whose operations are defined, for $\left\langle a_{1}, a_{2}\right\rangle,\left\langle b_{1}, b_{2}\right\rangle \in A \times A$, as follows:

$$
\begin{aligned}
\left\langle a_{1}, a_{2}\right\rangle \wedge\left\langle b_{1}, b_{2}\right\rangle & :=\left\langle a_{1} \sqcap b_{1}, a_{2} \sqcup b_{2}\right\rangle \\
\left\langle a_{1}, a_{2}\right\rangle \vee\left\langle b_{1}, b_{2}\right\rangle & :=\left\langle a_{1} \sqcup b_{1}, a_{2} \sqcap b_{2}\right\rangle \\
\left\langle a_{1}, a_{2}\right\rangle \otimes\left\langle b_{1}, b_{2}\right\rangle & :=\left\langle a_{1} \sqcap b_{1}, a_{2} \sqcap b_{2}\right\rangle \\
\left\langle a_{1}, a_{2}\right\rangle \oplus\left\langle b_{1}, b_{2}\right\rangle & :=\left\langle a_{1} \sqcup b_{1}, a_{2} \sqcup b_{2}\right\rangle \\
\left\langle a_{1}, a_{2}\right\rangle \supset\left\langle b_{1}, b_{2}\right\rangle & :=\left\langle\left(\sim a_{1}\right) \sqcup b_{1}, a_{1} \sqcap b_{2}\right\rangle \\
\neg\left\langle a_{1}, a_{2}\right\rangle & :=\left\langle a_{2}, a_{1}\right\rangle \\
\mathrm{f} & :=\langle 0,1\rangle \\
\mathrm{t} & :=\langle 1,0\rangle \\
\perp & :=\langle 0,0\rangle \\
\top & :=\langle 1,1\rangle \\
\square\left\langle a_{1}, a_{2}\right\rangle & :=\left\langle\square_{+} a_{1} \sqcap \square_{-} \sim a_{2}, \diamond_{+} a_{2}\right\rangle
\end{aligned}
$$

where $\diamond_{+} a_{2}:=\sim \square_{+} \sim a_{2}$. This construction is obviously related to (and to some extent generalizes) those of [24], [26], [23].

It can be directly checked that every twist-structure $\mathbf{A}^{\bowtie}$ satisfies the axioms of modal bilattices. With respect to the construction used in [24], [23] to represent so-called $B K$ lattices, we note that a twist-structure $\mathbf{A}^{\bowtie}$ is a BK-lattice precisely when the underlying bimodal Boolean algebra $\mathbf{A}$ satisfies the equation $\square_{-}(x)=1$, so that $\square\left\langle a_{1}, a_{2}\right\rangle=$ $\left\langle\square_{+} a_{1}, \diamond_{+} a_{2}\right\rangle$. It is also easy to check that

$$
\square\left(\left\langle a_{1}, a_{2}\right\rangle \vee\langle 0,0\rangle\right) \oplus\left(\square\left\langle a_{1}, a_{2}\right\rangle \wedge\langle 0,0\rangle\right)=\left\langle\square_{+} a_{1}, \diamond_{+} a_{2}\right\rangle
$$

which explains the relation between our modal operator and that of [23], [24] stated in Proposition 2. Obviously, our modal operator cannot be recovered as a term in the language of [24], because $\square$ is defined using two independent operators $\square_{+}$and $\square_{-}$on the underlying Boolean algebra, while [24] only makes use of one operator (together with its dual).

As with (non-modal) bilattices, it can be shown that every modal bilattice is isomorphic to a twist-structure. Given a modal bilattice $\mathbf{B}$, one first defines an (equivalence) relation $\approx$ by letting $a \approx b$ if $a \wedge b=a \oplus b$ [8, Definition 3.7]. One then shows that the quotient $\mathbf{B} / \approx$ can be endowed with algebraic operations that turn it into a bimodal Boolean algebra: for equivalence classes $[a],[b] \in B / \approx$ one lets

$$
\begin{aligned}
{[a] \sqcap[b] } & :=[a \wedge b] \\
{[a] \sqcup[b] } & :=[a \vee b] \\
\sim[a] & :=[a \supset \mathrm{f}] \\
\square_{+}[a] & :=[\diamond(a \supset \mathrm{f}) \supset \mathrm{f}] \\
\square_{-}[a] & :=[\square(\neg(a \supset \mathrm{f}) \vee \top)] \\
\diamond_{+}[a] & :=[\diamond a]
\end{aligned}
$$

where $\diamond$ abbreviates $\neg \square \neg$. We thus obtain the following representation:

Theorem 12. Every modal bilattice is isomorphic to the twiststructure $(\mathbf{B} / \approx)^{\bowtie}$ through the map $a \mapsto\langle[a],[\neg a]\rangle$ for $a \in B$. 
This result can be extended to an equivalence between categories corresponding to modal bilattices and twist-structures, as was done for non-modal bilattices in [19], [7].

This algebraic representation theorem further suggests that it ought to be possible to encode modal bilattice logic in classical modal logic (albeit one with two independent modalities). We leave this for further research.

\section{DUALITY}

In this section we develop a topological duality for modal bilattices based on the duality for bilattices introduced in [18], to which we also refer for further details and proofs.

Classical implicative bilattices, viewed as a category, are dually equivalent to a category of topological spaces that we called Stone bispaces (see [18, Theorem 3.16] and subsequent remarks). The two key ingredients for developing this duality are, firstly, that the $\langle\wedge, \vee, \supset, \neg, \mathrm{f}, \mathrm{t}\rangle$-reduct of a classical implicative bilattice is an N4-lattice [20], and, secondly, that the knowledge lattice operations are term-definable in the language $\langle\wedge, \vee, f, t, \perp, T\rangle$ (this is the so-called 90-degree lemma [18, Lemma 1.5]). A duality for classical implicative bilattices can thus be obtained by specializing the duality for N4-lattices introduced in [22]. We recall the relevant definitions and results.

Definition 13. A Stone bispace is a structure $\mathcal{X}=$ $\left\langle X, X^{1}, X^{2}, \tau, g\right\rangle$ such that:

(i) $\langle X, \tau\rangle$ is a Stone space

(ii) $X^{1}$ is closed in $\tau$ and $X^{2}=X \backslash X^{1}$

(iii) $g: \mathcal{X} \rightarrow \mathcal{X}$ is a homeomorphism such that $g^{2}=I d_{\mathcal{X}}$ and $g\left[X^{1}\right]=X^{2}$.

$A$ Stone bifunction $f: \mathcal{X} \rightarrow \mathcal{Y}$ between Stone bispaces $\mathcal{X}$ and $\mathcal{Y}$ is a continuous function such that:

(i) $f\left[X^{1}\right] \subseteq Y^{1}$

(ii) $f\left[X^{2}\right] \subseteq Y^{2}$

(iii) $f \circ g_{X}=g_{Y} \circ f$.

To any classical implicative bilattice $\mathbf{B}$ corresponds the Stone bispace $\left\langle X(\mathbf{B}), X^{1}(\mathbf{B}), X^{2}(\mathbf{B}), \tau_{\mathbf{B}}, g_{\mathbf{B}}\right\rangle$ where:

- $X(\mathbf{B})$ is the set of prime filters of the truth lattice $\langle B, \wedge, \vee, \mathrm{f}, \mathrm{t}\rangle$

- $\tau_{\mathbf{B}}$ is the topology generated by the sets $\Phi(x):=\{P \in$ $X(\mathbf{B}): x \in P\}$ for each $x \in B$

- $X^{1}(\mathbf{B}):=\Phi(\top)$

- $X^{2}(\mathbf{B}):=\Phi(\perp)$

- $g_{\mathbf{B}}(P):=\{x: \neg x \notin P\}$ for all $P \in X(\mathbf{B})$.

We stress the fact, as this will play a key role in our completeness proofs, that $X^{1}(\mathbf{B})$ is precisely the set of all prime bifilters of $\mathbf{B}$. Also note that the decomposition of $X$ into $X^{1}$ and $X^{2}$ naturally corresponds to the representation of classical implicative bilattices as a twist-structure, i.e., as a product of two Boolean algebras.

Conversely, given any Stone bispace $\mathcal{X}=\left\langle X, X^{1}, X^{2}, \tau, g\right\rangle$ we define a classical implicative bilattice structure on the set
$B(\mathcal{X})$ of clopens of $\tau$ with the operations defined as follows:

$$
\begin{array}{rlrl}
\mathrm{f} & :=\emptyset & \mathrm{t} & :=X \\
\top & :=X^{1} & \perp:=X^{2} \\
U \wedge V & :=U \cap V \\
U \vee V & :=U \cup V \\
U \otimes V & :=\left(U \cap \mathcal{X}^{2}\right) \cup\left(V \cap \mathcal{X}^{2}\right) \cup(U \cap V) \\
U \oplus V & :=\left(U \cap \mathcal{X}^{1}\right) \cup\left(V \cap \mathcal{X}^{1}\right) \cup(U \cap V) \\
U \supset V & :=\left(X^{1} \backslash(U \backslash V)\right) \cup\left(X^{2} \backslash(g(U) \backslash V)\right) \\
\neg U & :=X \backslash g(U) .
\end{array}
$$

These translations extend to morphisms in the usual way: To an algebraic homomorphism $h: \mathbf{B} \rightarrow \mathbf{B}^{\prime}$ corresponds a Stone bifunction $X(h): X\left(\mathbf{B}^{\prime}\right) \rightarrow X(\mathbf{B})$ defined by $X(h)(P):=h^{-1}[P]$ for all $P \in X\left(\mathbf{B}^{\prime}\right)$. Similarly, to a Stone bifunction $f: \mathcal{X} \rightarrow \mathcal{X}^{\prime}$ one associates a classical implicative bilattice homomorphism $B(f): B\left(\mathcal{X}^{\prime}\right) \rightarrow B(\mathcal{X})$ defined by $B(f)(U):=f^{-1}[U]$ for all $U \in B\left(\mathcal{X}^{\prime}\right)$. Thus we have:

Theorem 14. The category of Stone bispaces and Stone bifunctions and the category of classical implicative bilattices and algebraic homomorphisms are dually equivalent.

In order to establish a duality for modal bilattices note that by dropping $\square$ from the signature we obtain a classical implicative bilattice and thus the preceding theorem applies. On the other hand, if we consider the $\langle\wedge, \vee, f, t, \square\rangle$-reduct we obtain a distributive lattice with a finite-meet preserving operator for which a version of Jónsson-Tarski duality can be found in [17]. As we will see, the two dualities can be easily amalgamated into one.

Let us first recall how the duality in [17] works. To a distributive lattice $\mathbf{B}$ with a meet-preserving modal operator one associates the Priestley space $X(\mathbf{B})$ of prime filters where the topology is defined as usual, [11], and in particular as in the duality of Theorem 14. The order is just inclusion between prime filters. On $X(\mathbf{B})$ one defines a relation $R$ by

$$
\langle P, Q\rangle \in R \quad \text { iff } \quad \square^{-1}[P] \subseteq Q .
$$

It then follows that $R$ satisfies the following properties:

- $\subseteq ; R ; \subseteq=R$;

- $R[P]$ is a closed set for every $P \in X(B)$;

- $R^{-1}[D]$ is a clopen down-set for every clopen down-set $D \subseteq X(B)$.

Conversely, to any Priestley space $\langle X, \tau, \leq\rangle$ carrying a relation $R$ with above properties a distributive lattice with modal operator can be associated. This works as in the standard Priestley duality; elements of the dual are the clopen up-sets of $X$ and the lattice operations are the set-theoretic ones. The modal operator is defined by

$$
\square_{R} U:=\{x \in X: R[x] \subseteq U\} .
$$

For a modal bilattice $\mathbf{B}$ we can combine the dualities because the underlying set $X(\mathbf{B})$ for the dual is the same, i.e., the set of prime filters of the truth lattice. Since we know 
from Theorem 14 that $X(\mathbf{B})$ is actually a Stone space, we can simplify the properties of the induced relation $R$ to the following:

- $R[P]$ is a closed set for every $P \in X(B)$;

- $R^{-1}[U]$ is clopen for every clopen set $U \subseteq X(B)$.

The only outstanding issue is equation (iii) of Definition 7 which is not covered by either of the two constituent dualities. The following lemma not only remedies this situation but it will also be the crucial ingredient when we translate $X(\mathbf{B})$ to a four-valued Kripke structure.

Lemma 15. Let $\left\langle X(\mathbf{B}), X^{1}(\mathbf{B}), X^{2}(\mathbf{B}), \tau_{\mathbf{B}}, g_{\mathbf{B}}\right\rangle$ be the Stone bispace corresponding to a modal bilattice $\mathbf{B}$. Then the relation $R \subseteq X(\mathbf{B}) \times X(\mathbf{B})$ defined as in (5) satisfies the following properties:

- $\langle P, Q\rangle \in R$ iff $\langle g(P), g(Q)\rangle \in R$ for $P, Q \in X^{1}(B)$,

- $R\left[X^{2}(B)\right] \subseteq X^{2}(B)$.

It may be worth spelling out the two properties identified in the preceding lemma: (i) says that two points of $X^{1}$ are connected by $R$ iff the corresponding points in $X^{2}$ are connected; (ii) says that there cannot be a connection from $X^{2}$ to $X^{1}$.

Our analysis so far justifies the following definition:

Definition 16. A modal (Stone) bispace is a structure $\mathcal{X}=$ $\left\langle X, X^{1}, X^{2}, \tau, g, R\right\rangle$ such that:

(i) $\left\langle X, X^{1}, X^{2}, \tau, g\right\rangle$ is a Stone bispace;

(ii) $R[x]$ is a closed set for every $x \in X$;

(iii) $R^{-1}[U]$ is clopen for every clopen $U \subseteq X$;

(iv) $\langle x, y\rangle \in R$ iff $\langle g(x), g(y)\rangle \in R$ for $x, y \in X^{1}$;

(v) $R\left[X^{2}\right] \subseteq X^{2}$.

The above properties ensure that the clopens of a modal bispace form a modal bilattice when the $\square$ operator is defined as in (6). Thus we have established the object part of our duality between modal bispaces and modal bilattices. Notice also that properties (i)-(iii) in particular imply that $\langle X, \tau, R\rangle$ is a modal space (see, e.g., [4, Definition 3.1]).

In order to define a suitable notion of morphism for our spaces we simply adapt the definition of [17] to our special case.

Definition 17. A modal bifunction $f: \mathcal{X} \rightarrow \mathcal{Y}$ between modal bispaces $\mathcal{X}$ and $\mathcal{Y}$ is a Stone bifunction such that:

(i) if $\langle x, y\rangle \in R_{\mathcal{X}}$, then $\langle f(x), f(y)\rangle \in R_{\mathcal{Y}}$ for all $x, y \in X$;

(ii) if $\langle f(x), z\rangle \in R_{y}$, then there is $y \in X$ such that $f(y)=$ $z$, for every $x \in X$ and $z \in Y$.

Altogether we obtain:

Theorem 18. The category of modal bilattices with algebraic homomorphisms and the category of modal bispaces with modal bifunctions are dually equivalent.

\section{COMPLETENESS}

The duality of Theorem 18 provides us with a state-based semantics which, thanks to the algebraic completeness results stated in Section IV, can be proved to be complete with respect to our modal calculi. Let us sketch the proof for local consequence:

Assume $\Gamma \forall_{l} \varphi$. Then by Theorem 10 we know that there exists a matrix $\langle\mathbf{B}, F\rangle$, with $\mathbf{B}$ a modal bilattice and $F \subseteq B$ a bifilter of $\mathbf{B}$, and a homomorphism $h: \mathbf{F m} \rightarrow \mathbf{B}$ such that $h[\Gamma] \subseteq F$ but $h(\varphi) \notin F$. By the Prime Filter Theorem we can extend $F$ to a prime filter $P$ such that $h(\varphi) \notin P$. We then consider the modal bispace $\left\langle X(\mathbf{B}), X^{1}(\mathbf{B}), X^{2}(\mathbf{B}), \tau, g, R\right\rangle$ dual to $\mathbf{B}$ and the valuation $v$ from the set $F m$ of formulas to $B(X(\mathbf{B}))$, the set of clopen subsets of $X(\mathbf{B})$, defined by:

$$
v(\psi):=\{Q \in X(\mathbf{B}): h(\psi) \in Q\} .
$$

Note that $v$ is a homomorphism as it is the combination of $h$ with $\Phi$, the latter being the unit of the dual adjunction between modal bilattices and modal bispaces. For each element $Q$ of $X(\mathbf{B})$ we interpret the valuation as logical satisfaction in the usual way:

$$
Q \vDash \psi \text { iff } Q \in v(\psi) .
$$

By construction we have $P \vDash \gamma$ for all $\gamma \in \Gamma$ and $P \not \forall \varphi$, in other words, $\varphi$ does not follow semantically at point $P$, hence $\Gamma \not_{l} \varphi$.

This argument is correct (it is, after all, the standard argument one derives from Jónsson-Tarski duality for classical modal logic) but the overall set-up can be considered to be deficient in several ways. To start with, the valuation does not act homomorphically at each point of $X(\mathbf{B})$. We would expect, for example, to be able to say how $Q \vDash \neg \psi$ is derived from $Q \vDash \psi$ but of course this is not possible in the two-valued setting of the model. Similarly, the meaning of $\oplus$ and $\otimes$ differs depending on whether the point we consider is in $X^{1}(\mathbf{B})$ or in $X^{2}(\mathbf{B})$. We also observe that modal bispaces are not general Kripke structures, as the partition into two isomorphic halves is essential for the interpretation of formulas, and the relation must satisfy the conditions expressed in Lemma 15. Before we remedy the situation let us also point out one positive aspect of the bispace model, namely, that the interpretation of $\square$ is as one would expect: $Q \vDash \square \psi \Longleftrightarrow \forall\left\langle Q, Q^{\prime}\right\rangle \in R . Q^{\prime} \vDash \psi$.

Clearly, the mismatch between logic and model appears because the former is four-valued while the latter is twovalued in character. What we need to do, therefore, is to transform the model into a four-valued Kripke structure, which is in any case the desired semantic model. Our proof attempt gives us a hint as to how this should be done: a bifilter on a bilattice always contains the constant $T$, hence any prime extension will do so as well. This shows that the point $P$ constructed in the proof always belongs to $X^{1}(\mathbf{B})$ (see the text following Definition 13). Consequently, given a modal bispace $\mathcal{X}=\left\langle X, X^{1}, X^{2}, \tau, g, R\right\rangle$ we let $X^{1}$ be the carrier set of our four-valued Kripke structure. Then note that according to Definition 16, items (iv) and (v), the relation $R$ on $X$ is completely determined by two relations $R_{+}, R_{-}$on $X^{1}$, where

$$
\begin{aligned}
& \langle x, y\rangle \in R_{+} \text {iff }\langle x, y\rangle \in R ; \\
& \langle x, y\rangle \in R_{-} \text {iff }\langle x, g(y)\rangle \in R .
\end{aligned}
$$


In other words, $R_{+}$encodes the links internal to $X^{1}$ and $R_{-}$ those from $X^{1}$ to $X^{2}$. Definition 16 tells us that the links internal to $X^{2}$ are mirror images of those internal to $X^{1}$ and that there are no links from $X^{2}$ to $X^{1}$. We combine $R_{+}$and $R_{-}$ into a single four-valued relation $R_{4}: X^{1} \times X^{1} \rightarrow \mathcal{F O U R}$ as follows:

$$
R_{4}(x, y)=\left\{\begin{array}{cl}
\mathrm{t} & \text { iff }\langle x, y\rangle \in R_{+} \text {and }\langle x, y\rangle \in R_{-} \\
\top & \text { iff }\langle x, y\rangle \in R_{+} \text {and }\langle x, y\rangle \notin R_{-} \\
\perp & \text { iff }\langle x, y\rangle \notin R_{+} \text {and }\langle x, y\rangle \in R_{-} \\
\mathrm{f} & \text { iff }\langle x, y\rangle \notin R_{+} \text {and }\langle x, y\rangle \notin R_{-}
\end{array}\right.
$$

Standard duality theory tells us that the valuation $v$ in our proof attempt takes values in the bilattice $B(\mathcal{X})$ of clopen subsets of $X$. These, too, can be represented as subsets of $X^{1}$ alone, making use of the homeomorphism $g$ that exchanges $X^{1}$ and $X^{2}$. We $\operatorname{set}^{8}$

$$
\begin{aligned}
& v_{+}(\psi):=X^{1} \cap v(\psi) \\
& v_{-}(\psi):=g\left(X^{2} \backslash v(\psi)\right)
\end{aligned}
$$

As we did with $R_{+}$and $R_{-}$, we combine these two maps into a single four-valued valuation $v_{4}: F m \times X^{1} \rightarrow \mathcal{F O U} \mathcal{R}$ :

$$
v_{4}(\psi, x)=\left\{\begin{array}{cl}
\mathrm{t} & \text { iff } x \in v_{+}(\psi) \text { and } x \notin v_{-}(\psi) \\
\top & \text { iff } x \in v_{+}(\psi) \text { and } x \in v_{-}(\psi) \\
\perp & \text { iff } x \notin v_{+}(\psi) \text { and } x \notin v_{-}(\psi) \\
\mathrm{f} & \text { iff } x \notin v_{+}(\psi) \text { and } x \in v_{-}(\psi)
\end{array}\right.
$$

With these definitions we can now check that $v_{4}$ acts homomorphically at the points of $X^{1}$. For example:

$$
\begin{aligned}
v_{4}\left(\psi \wedge \psi^{\prime}, x\right)=\mathrm{t} & \\
& \Longleftrightarrow x \in v_{+}\left(\psi \wedge \psi^{\prime}\right) \text { and } x \notin v_{-}\left(\psi \wedge \psi^{\prime}\right) \\
& \Longleftrightarrow x \in v\left(\psi \wedge \psi^{\prime}\right) \text { and } g(x) \in v\left(\psi \wedge \psi^{\prime}\right) \\
& \Longleftrightarrow x \in v(\psi) \cap v\left(\psi^{\prime}\right) \text { and } g(x) \in v(\psi) \cap v\left(\psi^{\prime}\right) \\
& \vdots \\
& \Longleftrightarrow v_{4}(\psi, x)=\mathrm{t} \text { and } v_{4}\left(\psi^{\prime}, x\right)=\mathrm{t}
\end{aligned}
$$

We are ready to transform our proof from the beginning of this section into one for four-valued Kripke structures:

Theorem 19. For all $\Gamma \cup\{\varphi\} \subseteq F m$, the following are equivalent:

(i) $\Gamma \vdash_{l} \varphi$;

(ii) for every four-valued Kripke model $M=\langle W, R, v\rangle$ and every $w \in W$, it holds that $M, w \vDash \Gamma$ implies $M, w \vDash \varphi$.

Proof: The argument proceeds exactly as before but we now translate the modal bispace obtained from duality into a four-valued Kripke model as laid out above. Local satisfaction becomes:

$$
\begin{aligned}
Q \vDash \psi & \text { iff } Q \in v(\psi) \\
& \text { iff } Q \in v_{+}(\psi) \\
& \text { iff } v_{4}(\psi, Q) \in\{\mathrm{t}, \top\}
\end{aligned}
$$

since we know that $Q$ belongs to $X^{1}(\mathbf{B})$. We then have $P \vDash \gamma$ for all $\gamma \in \Gamma$ and $P \not \models \varphi$, in other words, $\Gamma \not \not_{l} \varphi$.

\footnotetext{
${ }^{8}$ The asymmetry between the two definitions being exactly the "twist" in the twist-structure construction of Section V.
}

Theorem 20. For all $\Gamma \cup\{\varphi\} \subseteq F m$, the following are equivalent:

(i) $\Gamma \vdash_{g} \varphi$;

(ii) for any four-valued Kripke model $M=\langle W, R, v\rangle$, if $M, w \vDash \Gamma$ for all $w \in W$, then $M, w \vDash \varphi$ for all $w \in W$.

Proof: Only a small adjustment is needed. By Theorem 9 we know that the algebraic countermodel is of the form $\left\langle\mathbf{B}, F_{0}\right\rangle$ where $F_{0}$ is the least bifilter of $\mathbf{B}$. Hence for all $Q \in X^{1}(\mathbf{B})$ we have that $Q \in v_{+}(\gamma)$ for every $\gamma \in \Gamma$, that is, $Q \vDash \Gamma$. On the other hand, the statement $P \not \models \varphi$ remains true, and we conclude that $\Gamma \not \forall_{g} \varphi$.

\section{CONCLUSIONS AND FURTHER WORK}

Belnap's four-valued logic is by now one of the standard candidates for dealing with missing or conflicting information. Through the work of many researchers, especially Ginsberg, Fitting, Arieli and Avron, it has been shown that the system has good mathematical properties and is a flexible and expressive tool for practical reasoning. We believe that the results presented here strengthen this argument further. The modal logic we obtain is very closely related to the classical case and only one further axiom, $\square(\perp \rightarrow \varphi) \leftrightarrow(\perp \rightarrow \square \varphi)$, is required to obtain an axiomatization that is complete with respect to fourvalued Kripke structures, the natural candidate for a semantics.

From a mathematical perspective, it is tempting to try to transfer other results from classical modal logic to the fourvalued case. For example, the logic we characterize is the minimal one, and it is natural to consider stronger axioms and their manifestation in Kripke structures. As mentioned at the end of Section V, it may be possible to embed modal bilattice logic in classical modal logic (with two independent modalities), and this may provide the basis for a systematic transfer of results and algorithms.

Research in applications of multi-valued logic to computer science and artificial intelligence has considered a number of bilattices other than $\mathcal{F O U} \mathcal{R}$. At this point in time we do not know whether our completeness result extends to such settings. The authors of [6] do better in this respect, though we can not use their results without adaptation as bilattices, viewed as residuated lattices, are not integral in the sense explained above. Also, the problem of axiomatizing the global consequence relation seems to be beyond the reach of the methods employed in [6].

Much progress has been made in recent years in modelling evolving systems as coalgebras and it has been found that the logic which is naturally associated with these (via duality) is modal in character. Given the canonical nature of our fourvalued modal logic it ought to be possible to extract it from a natural functor on the category of sets.

In computer science, classical modal logic has been extended in numerous ways and the resulting calculi have been employed in verification with great success. Still, large systems continue to pose a formidable challenge to verification tools and many ideas have been put forward to deal with this problem. Four-valued logic can be seen as a calculus for 
approximate reasoning, within which it is possible to leave some parameters unspecified or to continue the analysis even if some submodules have returned conflicting information. This appears to be an approach very well worth trying but it clearly requires the extension of our minimal calculus to richer modal languages. It should also be said that our particular (Hilbert-style) calculus, while complete, may not be suitable for automation and - as in the classical case alternative calculi may be required. We nevertheless hope that the techniques and results of this paper will prove to be a useful toolkit in such an endeavour.

\section{ACKNOWLEDGEMENTS}

We would like to thank the authors of [6] for providing the inspiration for the current study. We are in particular indebted to Félix Bou and Ramon Jansana for several helpful discussions during the initial stages of our research. Thanks are also due to Klaus Keimel who visited us during a crucial period of our work. He was supported by grant SC7-1112-20 of the London Mathematical Society. We are also grateful to the referees of LICS who provided a number of insightful and stimulating comments.

The first author was partially supported by grant EP/G041296/1 of the Engineering and Physical Sciences Research Council and the second by grant PIEF-GA-2010272737-BMDF of the Marie Curie programme of the European Union.

\section{REFERENCES}

[1] O. Arieli and A. Avron, "Reasoning with logical bilattices," Journal of Logic, Language and Information, vol. 5, no. 1, pp. 25-63, 1996.

[2] N. D. Belnap, "How a computer should think," in Contemporary Aspects of Philosophy, G. Ryle, Ed. Boston: Oriel Press, 1976, pp. 30-56.

[3] N. D. Belnap, Jr., "A useful four-valued logic," in Modern uses of multiple-valued logic (Fifth Internat. Sympos., Indiana Univ., Bloomington, Ind., 1975), J. M. Dunn and G. Epstein, Eds. Dordrecht: Reidel, 1977, pp. 5-37. Episteme, Vol. 2.

[4] N. Bezhanishvili and A. Kurz, "Free modal algebras: A coalgebraic perspective," in Proceedings of the 2nd International Conference (CALCO, 2007) held in Bergen, August 20-24, 2007, ser. Lecture Notes in Computer Science, T. Mossakowski, U. Montanari, and M. Haveraaen, Eds. Springer, 2007, vol. 4624, ch. 10, pp. 143-157.

[5] W. J. Blok and D. Pigozzi, Algebraizable logics, ser. Mem. Amer. Math. Soc. Providence: A.M.S., Jan. 1989, vol. 396.

[6] F. Bou, F. Esteva, L. Godo, and R. Rodríguez, "On the minimum manyvalued modal logic over a finite residuated lattice," Journal of Logic and Computation, vol. 21, no. 5, pp. 739-790, 2011.

[7] F. Bou, R. Jansana, and U. Rivieccio, "Varieties of interlaced bilattices," Algebra Universalis, vol. 66, no. 1, pp. 115-141, 2011.

[8] F. Bou and U. Rivieccio, "The logic of distributive bilattices," Logic Journal of the I.G.P.L., vol. 19, no. 1, pp. 183-216, 2011.

[9] A. Chagrov and M. Zakharyaschev, Modal Logic, ser. Oxford Logic Guides. Oxford University Press, 1997, vol. 35.

[10] J. Czelakowski, Protoalgebraic logics, ser. Trends in Logic-Studia Logica Library. Dordrecht: Kluwer Academic Publishers, 2001, vol. 10.

[11] B. A. Davey and H. A. Priestley, Introduction to lattices and order. Cambridge: Cambridge University Press, 1990.

[12] M. Fitting, "Many-valued modal logics," Fundamenta Informaticae, vol. 15, pp. 235-254, 1991.

[13] M. Fitting, "Many-valued modal logics, II," Fundamenta Informaticae, vol. 17, pp. 55-73, 1992.

[14] J. M. Font, "Belnap's four-valued logic and De Morgan lattices," Logic Journal of the I.G.P.L., vol. 5, no. 3, pp. 413-440, 1997.
[15] J. M. Font and R. Jansana, A general algebraic semantics for sentential logics, 2nd ed., ser. Lecture Notes in Logic. Springer-Verlag, 2009, vol. 7.

[16] N. Galatos, P. Jipsen, T. Kowalski, and H. Ono, Residuated Lattices: an algebraic glimpse at substructural logics, ser. Studies in Logic and the Foundations of Mathematics. Amsterdam: Elsevier, 2007, vol. 151.

[17] R. Goldblatt, "Varieties of complex algebras," Annals of Pure and Applied Logic, vol. 44, no. 3, pp. 173-242, 1989.

[18] A. Jung and U. Rivieccio, "Priestley duality for bilattices," Studia Logica, vol. 100, no. 1-2, pp. 223-252, 2012.

[19] B. Mobasher, D. Pigozzi, G. Slutzki, and G. Voutsadakis, "A duality theory for bilattices," Algebra Universalis, vol. 43, no. 2-3, pp. 109$125,2000$.

[20] S. P. Odintsov, "Algebraic semantics for paraconsistent Nelson's logic," Journal of Logic and Computation, vol. 13, no. 4, pp. 453-468, 2003.

[21] S. P. Odintsov, "On the representation of N4-lattices," Studia Logica, vol. 76, no. 3, pp. 385-405, 2004.

[22] S. P. Odintsov, "Priestley duality for paraconsistent Nelson's logic," Studia Logica, vol. 96, no. 1, pp. 65-93, 2010.

[23] S. P. Odintsov and E. I. Latkin, "BK-lattices. Algebraic semantics for Belnapian modal logics," Studia Logica, vol. 100, no. 1-2, pp. 319-338, 2012.

[24] S. P. Odintsov and H. Wansing, "Modal logics with Belnapian truth values," Journal of Applied Non-Classical Logics, vol. 20, pp. 279-301, 2010.

[25] U. Rivieccio, "An algebraic study of bilattice-based logics," Ph. D. Dissertation, University of Barcelona, 2010.

[26] U. Rivieccio, "Paraconsistent modal logics," Electronic Notes in Theoretical Computer Science, vol. 278, pp. 173-186, 2011.

[27] A. Tarski, "Grundzüge des Systemenkalküls," Fundamenta Mathematicae, vol. 25, pp. 503-526, 1935. 